\title{
Non-adiabatic description of proton emission from the odd-odd nucleus ${ }^{130} \mathrm{Eu}$
}

\author{
Monika Patial $^{1, a}$, P. Arumugam ${ }^{1}$, A.K. Jain ${ }^{1}$, E. Maglione ${ }^{2}$, and L.S. Ferreira ${ }^{3}$ \\ ${ }^{1}$ Department of Physics, Indian Institute of Technology Roorkee, Roorkee 247667, India \\ ${ }^{2}$ Dipartimento di Fisica e Astronomia "G. Galilei", Via Marzolo 8, I-35131 Padova, Italy and Istituto Nazionale \\ di Fisica Nucleare, Padova, Italy \\ ${ }^{3}$ Centro de Física das Interacções Fundamentais, and Departmento de Física, Instituto Superior Técnico, \\ Avenida Rovisco Pais, P1049-001 Lisbon, Portugal
}

\begin{abstract}
We discuss the non-adiabatic quasiparticle approach for calculating the rotational spectra and decay width of odd-odd proton emitters. The Coriolis effects are incorporated in both the parent and daughter wave functions. Results for the two probable ground states $\left(1^{+}\right.$and $\left.2^{+}\right)$of the proton emitter ${ }^{130} \mathrm{Eu}$ are discussed. With our calculations, we confirm the proton emitting state to be the $I^{\pi}=1^{+}$state, irrespective of the strength of the Coriolis interaction. This study provides us with an opportunity to look into the details of wave functions of deformed odd-odd nuclei to which the proton emission halflives are quite sensitive.
\end{abstract}

\section{Introduction}

Heavy nuclei $(Z>50)$ at the proton dripline are unbound to the emission of a constituent proton from their ground states. In a theoretical description within the adiabatic limit, it is assumed that decaying proton moves in the single-particle Nilsson level in resonance with the unbound core plus neutron system. However, it has been seen in the case of odd-even nuclei, the non-adiabatic effects play crucial role [1-3] and it will be interesting to extend these calculations to odd-odd nuclei. Adiabatic approach with zero point energy [4] has already explained some of the odd-odd nuclei, and we improve this approach by taking into account the finite moment of inertia, and hence the full rotational spectra, which allows considerable Coriolis mixing of quasiparticle states. This realistic approach along with the consideration of residual neutron-proton ( $n p$ ) interaction and Newby shift (for $K=0$ band) [5, 6], would lead to a precise study of the odd-odd proton emitters. Here we present the non-adiabatic approach along with proper treatment of quasiparticle mixing, to study the structure and decay of the proton rich odd-odd nuclei near the drip line. This formalism is applied to study proton emission from ${ }^{130} \mathrm{Eu}$ where we focus on the role of Coriolis interaction on the rotational states of the daughter nucleus ${ }^{129} \mathrm{Sm}$ and the decay width of ${ }^{130} \mathrm{Eu}$.

\footnotetext{
a e-mail: monikaphd@gmail.com
} 


\section{The non-adiabatic quasiparticle approach}

We consider a two quasiparticle plus rotor model [7,8] based on the mean field of Woods-Saxon potential [9] with deformed spin-orbital potential, to study the doubly odd proton emitting nucleus. The presence of residual pairing interactions are considered by the transformation of single-particle energy to quasiparticle energy through the relation $\tilde{\epsilon}_{k}=\sqrt{\Delta^{2}+\left(\epsilon_{k}-\lambda\right)^{2}}$, where $\lambda$ is the Fermi energy, $\epsilon_{k}$ is the single-particle energy and $\Delta$ is the pairing gap chosen as $12 / \sqrt{A}$. The complete Hamiltonian for the odd-odd nucleus can be expressed as sum of intrinsic part and rotational part where latter consists of sum of various terms including the Coriolis interaction between valence particles and collective rotation, the particle-particle coupling and the recoil term. We have introduced by a suitable constant value, the residual $n p$ interaction (GM splitting [5]) and the Newby shift [6]. We consider a variable moment of inertia (VMI) defined as $\mathfrak{I}(I)=\mathfrak{J}_{0} \sqrt{1+b I(I+1)}$, where $b$ is the VMI parameter and the constant $\mathfrak{I}_{0}$ is fixed such that $\frac{\hbar^{2}}{2 \mathfrak{I}(I=2)}=E_{2+}$, where the $E_{2+}$ is the first excited $2^{+}$level energy of the core. The symmetrized wave function for the parent nucleus takes the form as shown below:

$$
\Psi^{I, M}=\sum_{K_{p}, K_{n}, K_{T}=K_{n} \pm K_{p}} \sqrt{\frac{2 I+1}{16 \pi^{2}\left(1+\delta_{K_{T} 0}\right)}} a_{K_{T}, K_{n}, K_{p}}^{I}\left\{D_{M, K_{T}}^{I} \Upsilon_{K_{T}}^{K_{p}, K_{n}}+(-1)^{I+K_{T}} D_{M,-K_{T}}^{I} R_{i} \Upsilon_{K_{T}}^{K_{p}, K_{n}}\right\} .
$$

Here, $R_{i}$ is the rotational operator about second intrinsic axis. $a_{K_{T}, K_{n}, K_{p}}^{I}$ is the mixing coefficient obtained after diagonalising the total Hamiltonian matrix for the parent nucleus. Similarly, the mixing coefficient, $c_{K_{n}}^{I_{d}}$ for the daughter nucleus is obtained by solving the Hamiltonian for the rotor plus neutron system. Then the overlap is taken between the parent wave function and the tensorial product of daughter and emitted proton wave functions, which gives the partial decay width as written below:

$$
\Gamma_{l_{p} j_{p}}^{I, I_{d}}=\frac{\hbar^{2} \kappa}{\mu} \sum_{K_{n}, K_{p}\left(K_{T}=K_{n} \pm K_{p}\right)} a_{K_{T}, K_{n}, K_{p}}^{I} c_{K_{n}}^{I_{d}} \times\left.\sqrt{\frac{\left(2 I_{d}+1\right)}{(2 I+1)}}\left\langle I_{d}, K_{n}, j_{p}, K_{p} \mid I, K_{T}\right\rangle u_{K_{p}} N_{l_{p} j_{p}}^{K_{p}}\right|^{2},
$$

where $N_{l_{p} j_{p}}^{K_{p}}$ represents the asymptotic normalization factor. $u_{K_{p}}$ is the spectroscopic factor calculated from BCS approach and hence $u_{K_{p}}^{2}$ represents the probability of unoccupancy of proton level in daughter nucleus. The CG coefficients define angular momentum coupling between daughter and emitted proton. The total decay width is obtained by taking sum of partial decay widths from every possible combination of the angular momentum values of proton $\left(l_{p}\right.$ and $j_{p}$ values) and hence

$$
\Gamma^{I, J_{d}}=\sum_{j_{p}=\left|I-I_{d}\right|}^{I+I_{d}} \Gamma_{l_{p}, j_{p}}^{I, J_{d}}
$$

\section{Proton emission from ${ }^{130} \mathrm{Eu}$}

We studied the two probable ground states $\left(1^{+}\right.$and $\left.2^{+}\right)$of the highly deformed proton emitter ${ }^{130} \mathrm{Eu}$, for which adiabatic calculations were already reported in Ref. [10]. In Table 1, the quasiparticle levels for protons and neutrons in ${ }^{130} \mathrm{Eu}$, considered as basis states for Coriolis mixing, are shown at the predicted deformation $\beta_{2}=0.33$ by Möller and Nix [11]. The moment of inertia $\mathfrak{J}_{0}$ is evaluated by considering $E_{2+}$ of $122 \mathrm{keV}$ which is that of the nucleus ${ }^{130} \mathrm{Sm}$ and we have obtained the VMI parameter as 0.005. The mixing coefficients $a_{K_{T}, K_{n}, K_{p}}^{I}$, are obtained after diagonalising the total Hamiltonian where we have considered the mixing of above mentioned levels. The spin of ground state for the daughter nucleus is fixed by looking into minimum of energy for the combination of various spins 
Table 1. Nilsson orbitals chosen as basis for the Coriolis matrix, and corresponding quasiparticle energies at the deformation $\beta_{2}=0.33$, for protons and neutrons in the nucleus ${ }^{130} \mathrm{Eu}$.

\begin{tabular}{cccc}
\hline \multicolumn{2}{c}{ Protons } & \multicolumn{2}{c}{ Neutrons } \\
$\Omega\left[N n_{z} \Lambda\right]$ & $\tilde{\epsilon}_{k}(\mathrm{MeV})$ & $\Omega\left[N n_{z} \Lambda\right]$ & $\tilde{\epsilon}_{k}(\mathrm{MeV})$ \\
\hline $3 / 2[411]$ & 1.056 & $1 / 2[411]$ & 1.094 \\
$5 / 2[413]$ & 1.357 & $5 / 2[413]$ & 1.222 \\
$1 / 2[411]$ & 1.989 & $3 / 2[411]$ & 1.661 \\
$9 / 2[404]$ & 2.274 & $5 / 2[402]$ & 2.326 \\
\hline
\end{tabular}

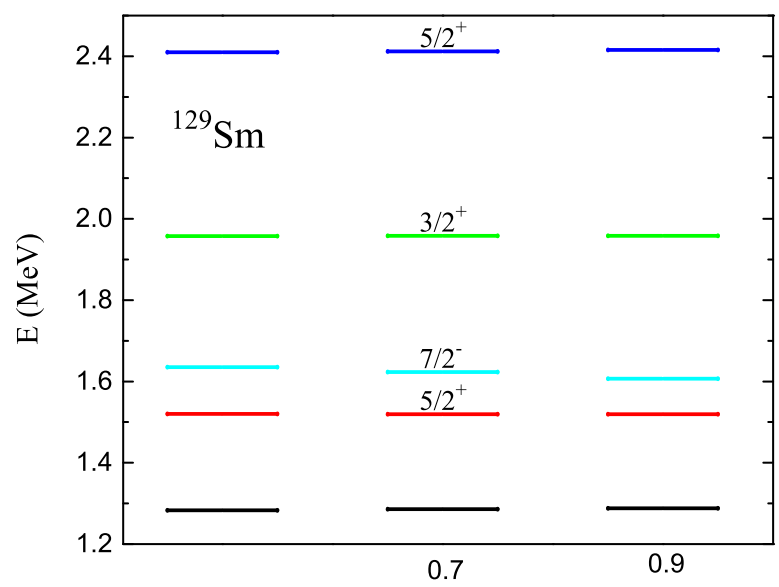

Figure 1. Rotational energy states at $\beta_{2}=0.33$ with different attenuation parameters for the daughter nucleus ${ }^{129} \mathrm{Sm}$.

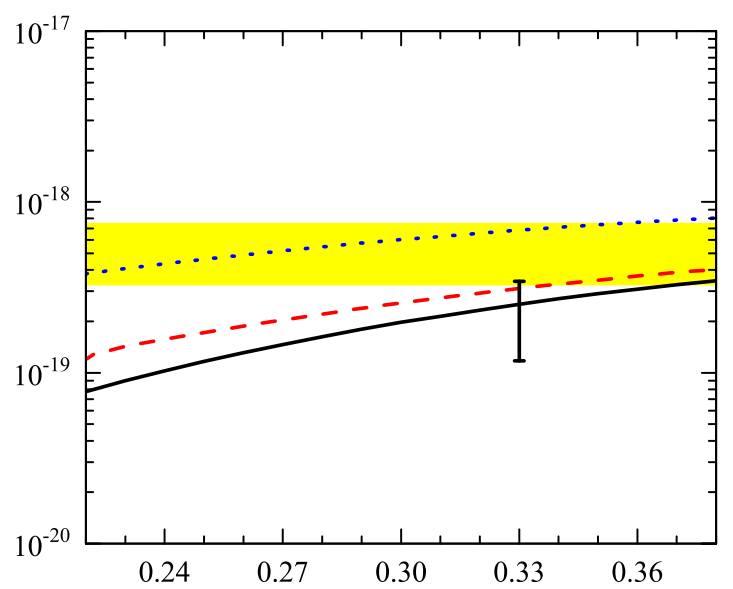

Figure 2. Decay width of ${ }^{130} \mathrm{Eu}$ for positive parity singlet $\left(K_{s}\right)$ and triplet $\left(K_{t}\right)$ states. The shaded yellow region represents the experimental values [10] of decay width. The error bar at the deformation $\beta_{2}=0.33$ is the uncertainty in calculations due to the error in the experimental $Q$-value. 
and parity. For ${ }^{129} \mathrm{Sm}$, the spin $1 / 2^{+}$always remains at lowest energy for different values of Coriolis attenuation parameter as can be seen in Figure 1. Hence it is considered that the ground state spin for ${ }^{129} \mathrm{Sm}$ is $1 / 2^{+}$. Decay widths of ${ }^{130} \mathrm{Eu}$ are then calculated for the singlet and triplet states without Coriolis interactions first and then the effect of Coriolis interaction is introduced with a $70 \%$ attenuation. Figure 2 shows the results for the both cases and we can see that the decay rate does not change with Coriolis attenuation for $I^{\pi}=1^{+}$state. Because of its low spin the higher $K$ orbitals cannot contribute and the low- $K$ states which can affect Coriolis mixing are far from the Fermi level. Clearly, it can be seen that the decay width corresponding to both the probable states $I^{\pi}=1^{+}, 2^{+}$are close to experimental value and hence any of them could be assigned as the proton emitting state. However, $I^{\pi}=1^{+}$is a triplet state, and is favoured by GM rule as lower in energy than the singlet state. Hence $I^{\pi}=1^{+}$is assigned to be the ground state of ${ }^{130} \mathrm{Eu}$ from which the proton is emitted. It is also clear that this conclusion does not depend on the attenuation of the Coriolis interaction.

\section{Acknowledgments}

This work was supported by the Fundação para a Ciência e a Tecnologia (Portugal), Project: CERN/FP/123606/2011. Authors (MP and AKJ) thank the CSIR and DST (Govt. of India) for providing the financial support.

\section{References}

[1] G. Fiorin, E. Maglione, L.S. Ferreira, Phys. Rev. C 67, 054302 (2003)

[2] P. Arumugam, L.S. Ferreira, E. Maglione, Phys. Lett. B 680, 443 (2009)

[3] P. Arumugam, L.S. Ferreira, E. Maglione, Phys. Rev. C 78, 041305 (2008)

[4] L.S. Ferreira, E. Maglione, Phys. Rev. Lett. 86, 1721 (2001)

[5] C.J. Gallagher, Jr., S.A. Moszkowski, Phys. Rev. 111, 1282 (1958)

[6] N.D. Newby, Phys. Rev. 125, 2063 (1962)

[7] A.K. Jain, R.K. Sheline, D.M. Headly, P.C. Sood, D.G. Burke, I. Hrǐvnácová, J. Kvasil, D. Nosek, R.W. Hoff, Rev. Mod. Phys. 70, 843 (1998)

[8] M. Patial, P. Arumugam, A.K. Jain, E. Maglione, L.S. Ferreira, Physics Letters B 718, 979 (2013)

[9] H. Esbensen, C.N. Davids, Phys. Rev. C 63, 014315 (2000)

[10] C.N. Davids, P.J. Woods, H. Mahmud, T. Davinson, A. Heinz, J.J. Ressler, K. Schmidt, D. Seweryniak, J. Shergur, A.A. Sonzogni et al., Phys. Rev. C 69, 011302 (2004)

[11] P. Moller, J. Nix, W. Myers, W. Swiatecki, At. Data Nucl. Data Tables 59, 185 (1995) 